Claremont Colleges

Scholarship@ Claremont

All HMC Faculty Publications and Research

HMC Faculty Scholarship

$1-1-1996$

\title{
The Steady Boundary Layer due to a Fast Vortex
}

Andrew J. Bernoff

Harvey Mudd College

Harald J. H. M. van Dongen

Northwestern University

Seth Lichter

Northwestern University

\section{Recommended Citation}

The steady boundary layer due to a fast vortex. Andrew J. Bernoff, Harald J. H. M. van Dongen, and Seth Lichter, Phys. Fluids 8,156 (1996).

This Article is brought to you for free and open access by the HMC Faculty Scholarship at Scholarship @ Claremont. It has been accepted for inclusion in All HMC Faculty Publications and Research by an authorized administrator of Scholarship @ Claremont. For more information, please contact scholarship@cuc.claremont.edu. 


\title{
The steady boundary layer due to a fast vortex
}

\author{
Andrew J. Bernoff \\ Department of Engineering Sciences and Applied Mathematics, Northwestern University, Evanston, \\ Illinois 60208 \\ Harald J. H. M. van Dongen and Seth Lichter ${ }^{\mathrm{a}}$ ) \\ Department of Mechanical Engineering, Northwestern University, Evanston, Illinois 60208
}

(Received 11 January 1995; accepted 13 September 1995)

\begin{abstract}
A point vortex located above and convected parallel to a wall is an important model of the process by which a boundary layer becomes unstable due to external disturbances. Often it has been assumed that the boundary layer due to the passage of the vortex is inherently unsteady. Here we show that for a vortex convected by a uniform shear flow, there is a steady solution when the speed of the vortex $c_{v}$ is sufficiently fast. The existence of the steady solution is demonstrated analytically in the limit of large vortex velocity $\left(c_{v} \rightarrow \infty\right)$ and numerically at more moderate speeds. This solution may provide a useful base state about which to investigate the stability of a boundary layer induced by external disturbances. (C) 1995 American Institute of Physics. [S1070-6631(96)01301-3]
\end{abstract}

\section{INTRODUCTION}

In this work we study steady boundary layer solutions due to the passage of a fast vortex near a wall. The vortex is "fast," in that it is convected by a shear flow at a speed that is much greater than the speed at which it would propagate due solely to induction by its image. The height of the vortex above the wall is fixed, as may reasonably be assumed from inviscid analysis (see Ref. 1) when the boundary layer thickness is small compared to the height of the vortex.

It has been previously observed that there are two general types of boundary layer response to the passage of a vortex. For slower vortices (including those propagating solely due to induction), reversed flow occurs in the near wall region, leading to a finite-time singularity in the solution to the boundary layer equations. Walker ${ }^{2}$ considered a vortex in a flow at rest at infinity and notes that for this situation there is no steady viscous solution. For this problem a singularity that forms in finite time was described by Elliot et $a l .{ }^{3}$ Walker's geometry was later considered with a Lagrangian numerical scheme ${ }^{4}$ and recently with an adaptive grid technique. ${ }^{5}$ In the present paper, the vortex is convected by a shear flow, a geometry previously considered by Doligalski et al. ${ }^{6}$ Their work focused on relatively slow moving vortices, and they concluded that the boundary layer will thicken locally in an "eruption" for arbitrary vortex speed. For faster vortices, though no temporal singularity has been observed, it has been suggested that the boundary layer grows continually in time. ${ }^{6}$ Doligalski et al. ${ }^{7}$ present an overview of the literature. Physical experiments relating to this problem are also reviewed in Luton et al. ${ }^{8}$

Note that in the case studied here, as in Ref. 6, the vortex is convected by a background flow of uniform shear. This shear is a solution to the equations of motion and so the boundary layer develops in response to the passage of the vortex alone. The case studied here is to be distinguished from those studies, e.g. Refs. 1 and 7, in which the boundary

${ }^{a)}$ Corresponding author: Tel: (708) 467-1885; FAX: (708) 491-3915; E-mail: s-lichter@nwu.edu. layer development is in response to both the passage of the vortex and an impulsively started flow.

Our goal here is to show that there exists a steady solution to the boundary layer equations for sufficiently fast vortices. Our numerics are not suited, nor do we try to pursue, the greater complexity of resolving the structure of separation. The existence of a steady solution for fast vortices is consistent with the work of Degani and Walker, ${ }^{9}$ who showed that for a rotating cylinder in a uniform flow, a sufficiently high rotation rate will suppress separation.

In the next section we formulate the problem and derive an asymptotic solution for the steady problem in the case of a weak boundary layer. Here, "weak" indicates that the fluid velocity is much smaller than the vortex velocity. This solution is valid both in the limit of large vortex speed and far downstream of the vortex, where the induced velocity is small. In Sec. III we describe a numerical formulation of the steady problem for finite vortex speed $c_{v}$. Numerical results are presented in Sec. IV, including plots of streamfunction, stagnation point location, wall shear stress, and displacement thickness. It is then shown that these measures of the numerical solution converge to the asymptotic solution in the limit of large vortex velocity $\left(c_{v} \rightarrow \infty\right)$. The implication of the existence of a steady solution and application of these results are discussed in Sec. V.

\section{FORMULATION}

We consider the boundary layer induced by a rectilinear potential vortex of strength $\tilde{\kappa}$, which can be positive or negative, at a height $y=H$ above a no-slip wall, Fig. 1 . The vortex is being advected by a linear shear profile, $u=\alpha y$ $(\alpha \geqslant 0)$, modeling an external flow; the shear velocity vanishes at the wall and consequently only affects the boundary layer structure indirectly through the advection of the vortex.

The equations of motion are nondimensionalized on the vortex height $H$ and twice the magnitude of the strength $2|\tilde{\kappa}|$. We consider the limit of large Reynolds number,

$$
\operatorname{Re}=\frac{2|\tilde{\kappa}|}{\nu},
$$



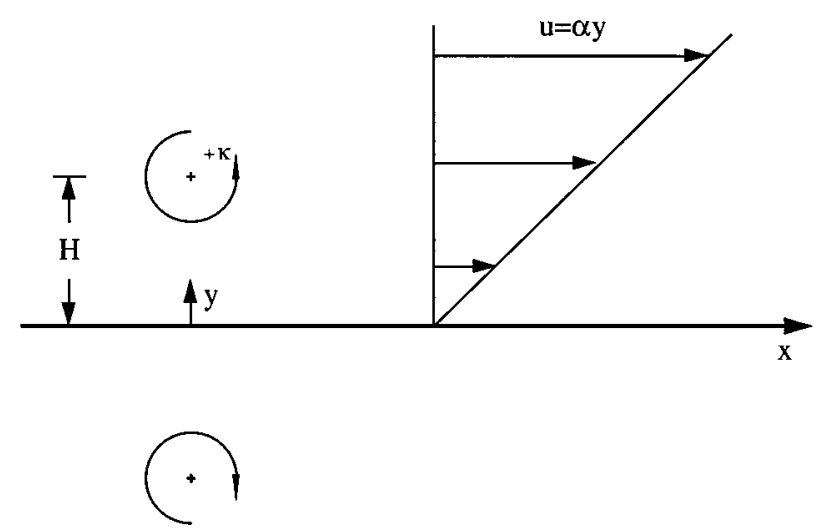

FIG. 1. Schematic of the geometry in dimensional variables. A vortex, here of strength $+\kappa$, is located a distance $y=H$ above a wall. An image vortex is equidistant below the wall at the same $x$ location. The vortex is convected by a shear flow $u=\alpha y$.

where $\nu$ is the kinematic viscosity. In the nondimensional coordinate system, a vortex of strength $\kappa= \pm \frac{1}{2}$ at unit height is advected by a shear $u=a y$, where $a=H^{2} \alpha / 2 \tilde{\kappa}$.

The inviscid problem can be solved by considering an image vortex, (cf. Fig. 1), of opposite sign symmetrically placed relative to the wall. Consequently, the vortex will propagate parallel to the wall with a velocity $c_{v}$ equal to the velocity induced by the vortex's image in the wall $\left(c_{i}= \pm \frac{1}{4}\right)$, together with the advection due to the imposed shear $\left(c_{s}=a\right)$,

$$
c_{v}=c_{s}+c_{i}=a \pm \frac{1}{4} .
$$

In the case in which the vortex strength is positive, the vortex and the shear flow both induce a positive velocity; in this case $c_{v}>\frac{1}{4}$. When the vortex strength is negative, the negative velocity induced by the image vortex is counter to the shear flow; in this case $c_{v}>-\frac{1}{4}$.

The vortex and its image, together with the background shear, create an inviscid velocity field of the form

$$
\begin{gathered}
(\bar{u}, \bar{v})=\left(a y \pm \frac{\left(x-c_{v} t\right)^{2}-y^{2}+1}{\left[\left(x-c_{v} t\right)^{2}+y^{2}+1\right]^{2}-4 y^{2}},\right. \\
\\
\left.\frac{ \pm 2\left(x-c_{v} t\right) y}{\left[\left(x-c_{v} t\right)^{2}+y^{2}+1\right]^{2}-4 y^{2}}\right) .
\end{gathered}
$$

Although this velocity field satisfies the impermeability condition, it does not satisfy the no-slip condition. Consequently, a boundary layer will form near the wall. A standard boundary layer scaling is now made,

$$
\begin{aligned}
& T=t, \quad X=x-c_{v} t, \quad Y=\sqrt{\operatorname{Re}} y, \\
& U(X, Y, T)=u(x, y, t), \quad V(X, Y, T)=\sqrt{\operatorname{Re}} v(X, Y, T) .
\end{aligned}
$$

The pressure in the boundary layer is independent of height at leading order; evaluating it in the inviscid region and substituting yields the boundary layer equations,

$$
\frac{\partial U}{\partial X}+\frac{\partial V}{\partial Y}=0
$$

$$
\frac{\partial U}{\partial T}+\left(U-c_{v}\right) \frac{\partial U}{\partial X}+V \frac{\partial U}{\partial Y}=\left(\bar{U}-c_{v}\right) \frac{d \bar{U}}{d X}+\frac{\partial^{2} U}{\partial Y^{2}} .
$$

The velocity $(U, V)$ must match the inviscid solution (2) outside the boundary layer $Y \gg 1$ but still near the wall $y \ll 1$. That is,

$$
(U, V) \rightarrow(\bar{U}, \bar{V}), \quad \text { as } \quad Y \rightarrow \infty,
$$

where

$$
(\bar{U}, \bar{V})=\left( \pm \frac{1}{X^{2}+1}, \pm \frac{2 X Y}{\left(X^{2}+1\right)^{2}}\right) .
$$

Note that the boundary layer is much thinner than the characteristic scale of the shear layer. Consequently, the shear has no direct role in boundary layer development to leading order and acts only to convect the vortex.

In addition, the no-slip condition must be satisfied,

$$
U(X, 0, T)=V(X, 0, T)=0 .
$$

To complete the formulation of the problem we must also prescribe initial conditions on the velocity. We will assume that the fluid initially has the inviscid profile,

$$
U(X, Y, 0)=\bar{U}, \quad V(X, Y, 0)=\bar{V},
$$

as if the vortex were turned on at $t=0+$. We believe this condition is appropriate for a vortex propagating into undisturbed shear flow, as any initial disturbance will eventually be far upstream of the vortex. Moreover, as we are most concerned with a class of steady solutions this condition has little effect on the subsequent analysis.

We will concentrate our efforts on finding a steady solution to the boundary layer equations in the frame moving with the vortex; to this end we note that if the term $\left(U-c_{v}\right)$ is negative, corresponding to flow in the upstream direction, the steady problem will be parabolic in nature and we can, at least in theory, solve the problem by integrating from downstream $x=\infty$ to upstream $x=-\infty$. This condition requires that

$$
c_{v}>\max (U),
$$

where $\max (U)$ is the maximum value of the velocity in the boundary layer. Where this condition is violated, there will be a region of upstream flow, and the strictly parabolic nature of the equations is lost; the question of existence of a steady solution becomes much more delicate in this case.

\section{A. Weak boundary layer approximation}

The boundary layer equations imply that the viscous perturbation of the velocity field from the inviscid velocity profile (8) is localized near the boundary. If, in addition, the fluid velocity is assumed to be small compared to the vortex velocity an approximate solution can be found. The boundary layer response is then weak, and the governing equations can be linearized around the inviscid solution. This approximation is valid in two regimes: first, when the speed of propagation of the vortex is large $\left(c_{v} \gg 1\right)$ and the cumulative response at any fixed spatial location is small due to the fast passage of the vortex; and second, far downstream from the vortex where the quiescent fluid only feels the weak algebraic precursors of the vortex induced velocity. 
To linearize the equations around the inviscid profiles, define perturbation velocity components $(k, l)$,

$$
U=\bar{U}+k(X, Y, T), \quad V=\bar{V}+l(X, Y, T) .
$$

If we assume that the fluid velocity is much less than the vortex velocity $\left(|U|,|V| \ll c_{v}\right)$, then, at leading order, the perturbation velocity satisfies the linearized boundary layer equations,

$$
\begin{aligned}
& \frac{\partial k}{\partial X}+\frac{\partial l}{\partial Y}=0, \\
& \frac{\partial k}{\partial T}-c_{v} \frac{\partial k}{\partial X}=\frac{\partial^{2} k}{\partial Y^{2}} .
\end{aligned}
$$

We expect the perturbation velocity to vanish in the farfield $Y \gg 1$. At the boundary, the no-slip condition must be satisfied,

$$
k(X, 0, T)=\frac{\mp 1}{X^{2}+1}, \quad l(X, 0, T)=0 .
$$

The initial condition (10) indicates that both components of the perturbation velocity initially vanish.

Note that the linearized momentum equation (14) decouples from the incompressibility equation (13) and takes the form of a diffusion equation for the horizontal velocity perturbation $k$, with a source term (15) on the boundary. The incompressibility equation now yields a parasitic definition for the vertical velocity perturbation,

$$
l(X, Y, T)=-\int_{0}^{Y} \frac{\partial k\left(X, Y^{\prime}, T\right)}{\partial X} d Y^{\prime}
$$

A steady solution to the diffusion equation for $k$ is derived below.

It is possible to write down a time-dependent solution to the diffusion equation for $k(14)$ by interpreting the boundary condition (15) as a source on the boundary and using a Green's function formulation. ${ }^{10}$ However, as the source term is integrable, we expect that in the frame moving with the vortex the solution will rapidly approach a steady velocity distribution in the neighborhood of the vortex and leave the initial transient behind at a fixed point in the laboratory frame. Moreover, as the dynamics in this limit are governed by the diffusion equation, we expect that this solution will be stable and attracting for all initial conditions that are undisturbed downstream of the vortex. Consequently, we limit our study to this steady response.

The steady solution for $k$ takes the form

$$
k(X, Y, T)=\mp K(X, \eta), \quad \eta=\sqrt{c_{v}} Y,
$$

and satisfies the steady diffusion equation

$$
\begin{aligned}
& K_{X}+K_{\eta \eta}=0, \\
& K(X, 0)=\frac{1}{X^{2}+1}, \\
& \lim _{\eta \rightarrow \infty} K(X, \eta)=0 .
\end{aligned}
$$

A solution can be found by using a Green's function ${ }^{10}$ formulation on the boundary

$$
K(X, \eta)=\frac{\eta}{2 \sqrt{\pi}} \int_{0}^{\infty} \frac{e^{-\eta^{2} / 4 \xi}}{\xi^{3 / 2}\left[1+(X+\xi)^{2}\right]} d \xi,
$$

which can be transformed to a more useful form by the change of variables $r=\eta / \sqrt{2 \xi}$,

$$
K(X, \eta)=\sqrt{\frac{2}{\pi}} \int_{0}^{\infty} \frac{r^{4} e^{-r^{2} / 2}}{r^{4}+\left(X r^{2}+\eta^{2} / 2\right)^{2}} d r .
$$

This integral form of the solution can be used to derive a number of useful results, leading to a description of the flow in the boundary layer.

We expect that the perturbation velocity will be weak far downstream $(X \gg 1)$; in this region the solution can be expanded in terms of similarity variables,

$$
K(X, \eta)=\frac{g(\zeta)}{X^{2}}+\mathscr{Q}\left(X^{-4}\right), \quad \zeta=\frac{\eta}{\sqrt{2 X}},
$$

where

$$
g(\zeta)=\sqrt{\frac{2}{\pi}} \int_{0}^{\infty} \frac{r^{4} e^{-r^{2} / 2}}{\left(r^{2}+\zeta^{2}\right)^{2}} d r .
$$

Note that $g$ is a monotonically decreasing function of $\zeta$. For small $\zeta$, corresponding to being near the solid boundary, $g(\zeta) \sim 1$, which recovers the no-slip boundary condition. For large $\zeta$, the integral can be expanded to yield

$$
g(\zeta) \sim \frac{1}{\zeta^{4}}, \quad K(X, \eta) \sim \frac{4}{\eta^{4}}+\odot\left(\frac{X}{\eta^{2}}\right) .
$$

Physically $|g| \leqslant 1$ implies $|K|<1 / X^{2}$. So the boundary layer is becoming weaker as $X \rightarrow \infty$.

The solution (20) is also uniformly valid in the limit of large vortex velocity $\left(c_{v} \gg 1\right)$; this can be verified by substituting the scaling (17) into the momentum equation (6) and retaining the leading-order terms. In Sec. IV we compare the results in the large-velocity limit to numerical calculations over a range of $c_{v}$.

In this limit, linearizing the governing equations (13)(15) about the steady solution yields a homogeneous diffusion equation. This suggests that the solution is stable to small perturbations.

\section{B. Streamfunction}

The streamfunction $\psi(X, Y)$ for the velocity satisfies

$$
\left(\psi_{Y},-\psi_{X}\right)=(U, V)
$$

and can be most easily constructed from our solutions by specifying that $\psi=0$ on the solid boundary, which yields

$$
\psi(X, Y)=\int_{0}^{Y} U\left(X, Y^{\prime}\right) d Y^{\prime} .
$$




$$
\psi(X, Y) \equiv \frac{\bar{\psi}(X, \eta)}{\sqrt{c_{v}}}
$$

which in the weak boundary layer limit approaches

$$
\lim _{c_{v} \rightarrow \infty} \bar{\psi}(X, \eta)= \pm \frac{\eta}{1+X^{2}} \mp \int_{0}^{\eta} K(X, \eta) d \eta .
$$

\section{Displacement thickness}

Two useful measures of the boundary layer considered are the displacement thickness and the wall stress, both of which can be computed analytically from the integral representation for large $c_{v}$.

The displacement thickness of the boundary layer can be defined as

$$
\delta^{*}(X)=-\frac{\int_{0}^{\infty} k\left(X, Y^{\prime}\right) d Y^{\prime}}{\bar{U}(X)} .
$$

It is convenient to introduce a scaled displacement thickness,

$$
\delta^{*}(X)=\sqrt{\frac{\pi}{2 c_{v}}} \bar{\delta}^{*}(X)
$$

In the limit of large $c_{v}$, this can be computed by substituting the integral formulation (20) and interchanging the order of integration,

$$
\lim _{c_{v} \rightarrow \infty} \bar{\delta}^{*}(X)=\left(\frac{1+X^{2}}{\sqrt{1+X^{2}}+X}\right)^{1 / 2} .
$$

Note that the scaled thickness $\bar{\delta}^{*}(X)$ is independent of vortex speed and hence should provide a uniformly valid approximation for the displacement thickness in the limit of high speed.

\section{Wall stress}

The wall stress is proportional to $U_{Y}(X, 0)$,

$$
\tau_{w}(X)=\mu U_{Y}(X, 0)=\mu \sqrt{\frac{\pi c_{v}}{2}} \bar{\tau}_{w}(X),
$$

where $\bar{\tau}_{w}(X)$ is the wall shear stress scaled with respect to the vortex speed. In the weak boundary layer limit $\left(c_{v} \rightarrow \infty\right)$, the scaled wall stress approaches

$$
\bar{\tau}_{w}(X)= \pm \frac{2 X+\sqrt{1+X^{2}}}{2\left(1+X^{2}\right)^{3 / 2} \sqrt{X+\sqrt{1+X^{2}}}},
$$

where the sign of $\bar{\tau}$ matches that of the vortex.

A stagnation point appears on the boundary and can be located at the point $X=X_{\text {stag }}$, where $\tau_{w}$ passes through zero. In the limit of large $c_{v}$, Eq. (32) yields $X_{\text {stag }}=-1 / \sqrt{3}$.

\section{NUMERICAL FORMULATION AND PARABOLICITY}

To solve the boundary layer equations numerically, we use the Davis Coupled Scheme ${ }^{11}$ which leads to a secondorder accurate solution in the grid spacings $\Delta X, \Delta Y$. The method assumes the local parabolicity of momentum equation. The basic strategy is to assume that the weak boundary layer solution is valid at some large $X$, and then to integrate in the upstream direction. At each point in $X$ we apply noslip boundary conditions at the wall $(Y=0)$, and assume Neumann boundary conditions on the deviation of $U$ from the inviscid flow $(\bar{U})$ at some large value of $Y$. At each step this implicit scheme requires the inversion of a pentadiagonal matrix to integrate a distance $\Delta X$ upstream. If at any point $U-c_{v}$ becomes positive, corresponding to a loss of local parabolicity [cf. Eq. (11)], the scheme will become unstable to short waves. Typically, we have chosen $(\Delta X, \Delta Y)$ as small as $(-0.001,0.005)$ and a computational domain of $X=[-12.5,12.5], Y=[0,25]$; refinement studies verified that our results are insensitive to numerical parameters.

Numerical solutions can be expected where the criterion (11) is everywhere satisfied. For $\kappa=\frac{1}{2}$ numerical solutions were found for $c_{v} \geqslant 1$. This can be understood by noting that the maximum value of $U$ is apparently 1 , obtained at $X=0$ in the matching region, where $U$ is given by $\bar{U}$ [cf. Eq. (8)]. For $\kappa=-\frac{1}{2}$, solutions were also found for $c_{v} \geqslant 1$. This can be understood by noting that the velocity becomes positive in a region upstream from the vortex [cf. Fig. 6]. The exact value of $c_{v}$ at which parabolicity was lost was not computed because of the stiffness of the calculations as $c_{v}$ decreases.

Below we present the results of our numerical calculations and compare them to the asymptotic results found in the limit of large velocity.

\section{RESULTS}

\section{A. Streamfunction}

Figure 2 shows the streamlines as a function of the spatial variables $(X, \eta)$. The interval between contour levels of streamfunction is 0.1 with non-negative values shown as solid lines and negative values as dashed lines. Recall that the vertical scaling $\eta$ contains the square root of the speed of the vortex (17) as well as the Reynolds number (3). So the figures greatly exaggerate the vertical scale in order to reveal the details of the boundary layer flow. The results of Sec. II A for $\kappa=\frac{1}{2}$ as $c_{v} \rightarrow \infty$ are presented in Fig. 2(c). For positive $X$, the flow closely approximates the inviscid flow field $(\bar{U}, \bar{V})$ which is a family of parabolas symmetric with respect to $X=0$, [cf. Eq. (8)]. For negative $X$, however, the upstream portion of these streamlines are concentrated into a narrowband, resulting in a downward jet-like flow. In addition, for negative $X$, there is a wedge-shaped region of reverse flow. The reverse flow region is separated from the region of forward flow by a stagnation streamline that intersects the wall at $X=-1 / \sqrt{3}$, as can be computed by setting $\tau_{w}=0$ in Eq. (32). For a negative vortex $\kappa=-\frac{1}{2}$, the pattern of streamlines is identical but the direction of flow is reversed.

The flow field for finite speeds $c_{v}$ are shown in Figs. 2(a) and 2(b). Figure 2(a) is for a speed $c_{v}=2.0$. Figure 2(b) is for a high speed of vortex propagation $c_{v}=10.0$. Note that as the vortex speed increases, the flow pattern approaches that shown for $c_{v} \rightarrow \infty$, Fig. 2(c). The streamline patterns for a negative vortex $\kappa=-\frac{1}{2}$ are comparable to the positive vortex results at the same speed with the reversed direction of flow. 


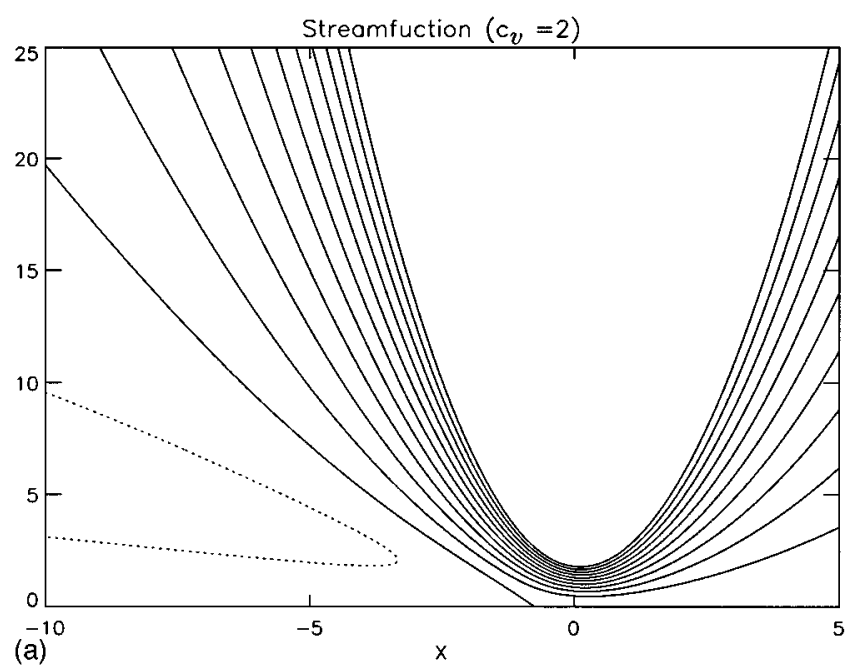

(a)

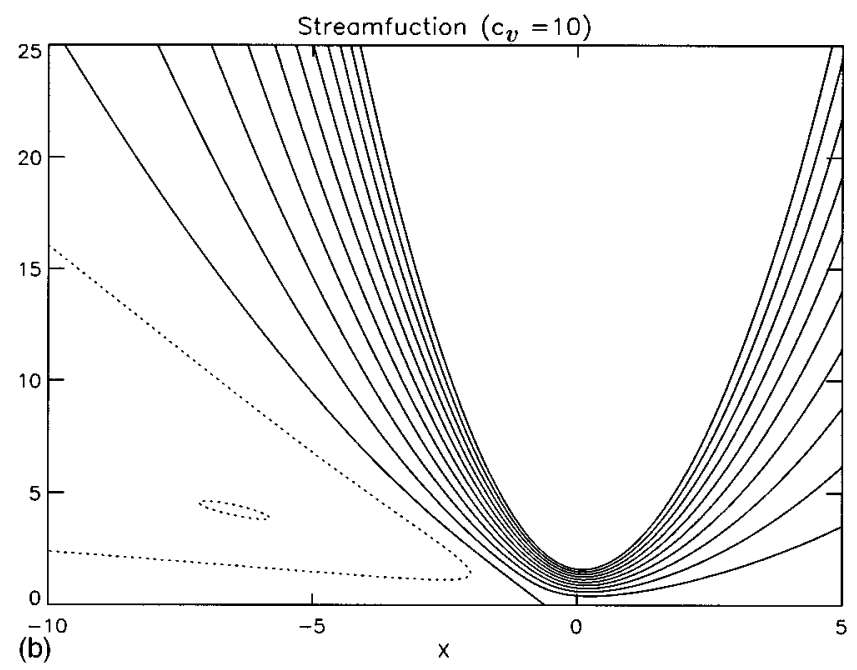

$\left(\right.$ (b) $^{-10}$

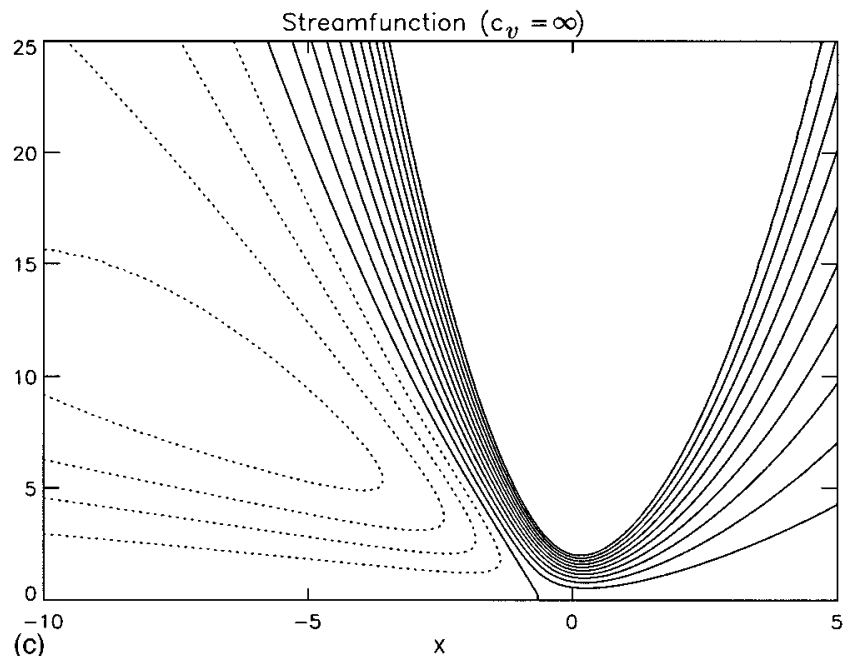

(c)
Stagnation Point Location

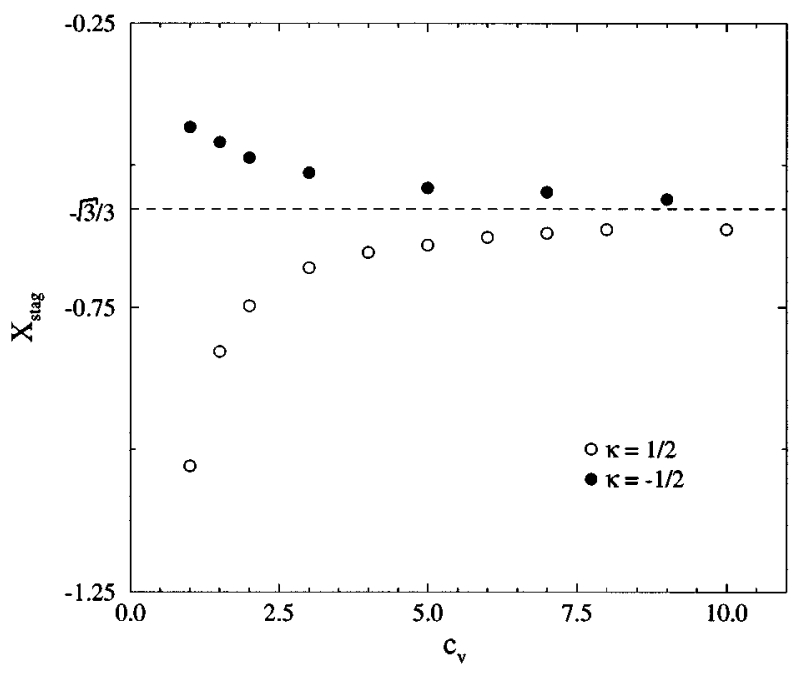

FIG. 3. The location of the stagnation point $X_{\text {stag }}$ as a function of the speed $c_{v}$ of the vortex. For vortices of both positive $\kappa=\frac{1}{2}$ and negative $\kappa=-\frac{1}{2}$ strength, the location of the stagnation point approaches $-1 / \sqrt{3}$, the asymptotic value in the limit $c_{v} \rightarrow \infty$; see Sec. IV A.

\section{B. Stagnation streamline}

For all speeds studied, there is one stagnation streamline. This streamline intersects the wall at some location $X_{\text {stag }}$, Fig. 3. As the vortex speed $\left|c_{v}\right|$ becomes large, the value of $X_{\text {stag }}$ approaches the asymptotic value of $-1 / \sqrt{3}$. For $\kappa=\frac{1}{2}$ $\left(\kappa=-\frac{1}{2}\right)$ the flow along the stagnation streamline is toward (away from) the wall. As the speed of the vortex slows, $X_{\text {stag }}$ moves upstream (downstream).

\section{Shear stress}

Figures 4(a) and 4(b) show the shear stress distribution along the wall for the positive and negative vortex cases, respectively. On both figures, the infinite velocity case is given by the solid line. As the vortex speed $\left|c_{v}\right|$ becomes large, the shear stress approaches that given by the infinite velocity vortex. The shear stress is small except in the interval around the vortex location. For $\kappa=\frac{1}{2}$ and $X \gg 0$ the shear stress is small and positive. Recall that in this region, the flow is well approximated by the infinite velocity results, and so the shear stress indicates that only a weak boundary layer has been stirred up by the algebraically small $1 /\left(X^{2}+1\right)$ vortex velocity. For $X<0$ the negative values of the stress are indicative of the reverse flow region. Note that the shear stress is zero at the stagnation point shown in Fig. 3. On reflecting the $\kappa=\frac{1}{2}$ results about the ordinate, one finds the qualitative behavior for $\kappa=-\frac{1}{2}$. The values, however, are not precisely reproduced, as changing the sign of $\kappa$ does not precisely reverse the sign of the problem statement-the vortex always convects toward $X \rightarrow \infty$.

\section{Displacement thickness}

The displacement thickness, defined in Eq. (30), normalized with respect to $\bar{U}$ is shown in Figs. 5(a) and 5(b) for positive and negative values of vortex strength $\kappa= \pm \frac{1}{2}$, re- 
Shear Stress

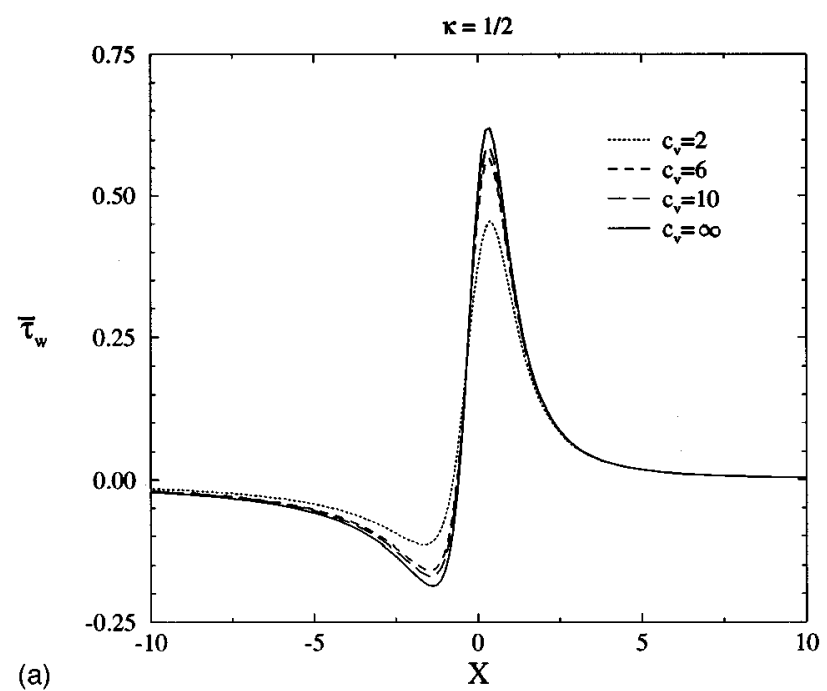

Shear Stress

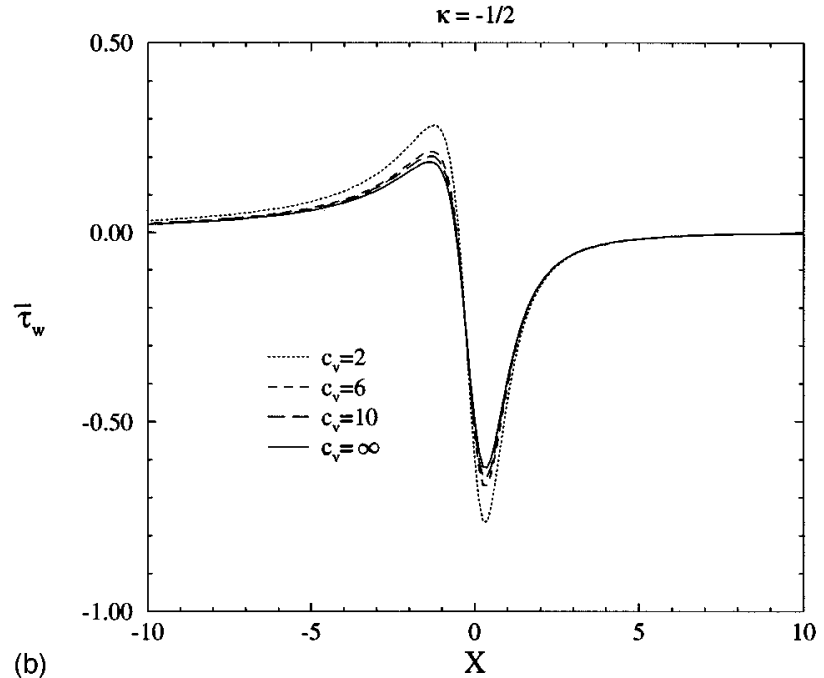

(b)

FIG. 4. Shear stress $\bar{\tau}_{w}$ as a function of distance $X$ along the wall. The vortex is located at $X=0$. As the speed of the vortex $c_{v}=2,6,10$ increases, the shear stress distribution approaches that given by the asymptotic result $c_{v} \rightarrow \infty$. Note that, as suggested by the linearized equation (14), the results for (a) vortices of positive strength $\kappa=\frac{1}{2}$ and (b) vortices of negative strength $\kappa=-\frac{1}{2}$ are nearly mirror images of one another; increasingly so as $c_{v} \rightarrow \infty$. (b) For the caption see the previous figure.

spectively. For $X \gg 0$ the normalized displacement thickness is very thin-another manifestation of the high speed of the vortex; viscous diffusion from the wall does not have a long time to act before the arrival of the vortex. Upstream, for $X \ll 0$, the values decay slowly; the smallest value of $\bar{U} \bar{\delta}^{*}$ shown for positive $X$ (at $X=10.0)$ is only reached at $X \approx$ -40 .

In both figures the solid line represents the asymptotic result as $c_{v} \rightarrow \infty$. From Eq. (30) it can be determined that the maximum thickness of $\bar{U} \bar{\delta}^{*}$ occurs at $X=-1 / \sqrt{3}$. For finite vortex speed, the maximum normalized thickness occurs for $X<0$ and approaches the limiting value as vortex speed increases. As $c_{v}$ increases the general shape of the displace-

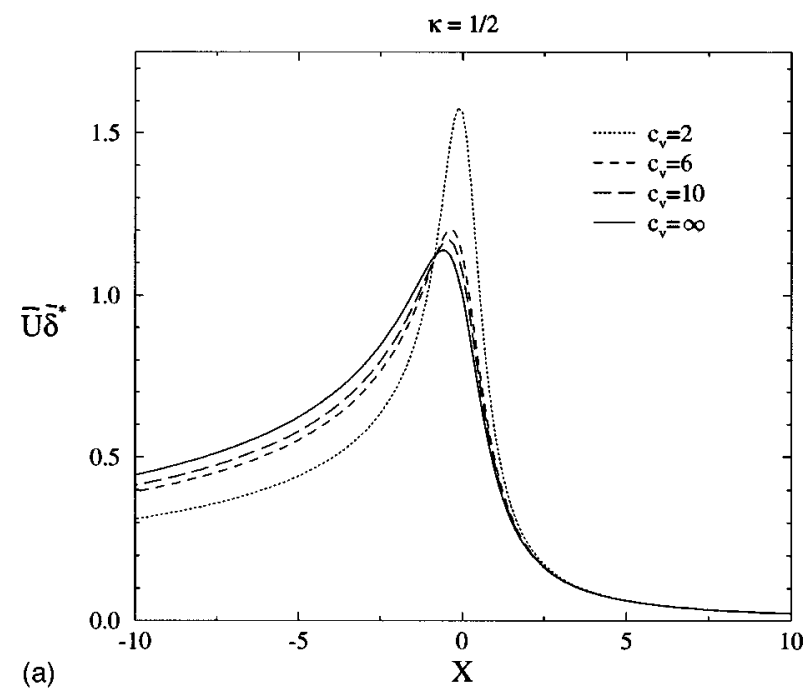

Displacement Thickness

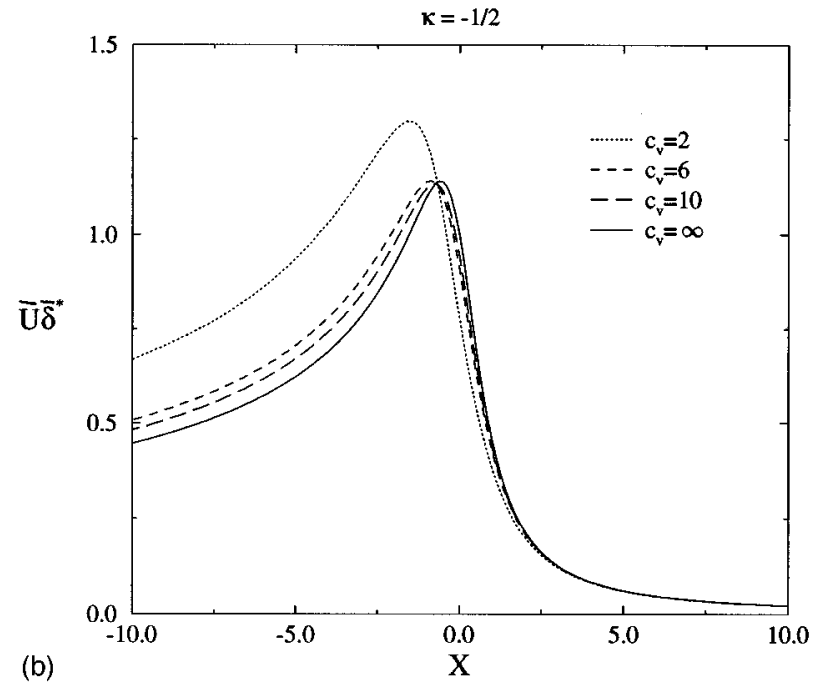

FIG. 5. The displacement thickness $\bar{\delta}^{*}$ normalized by the free-stream velocity at the edge of the boundary layer $\bar{U}$ as a function of distance $X$ along the wall. The vortex is located at $X=0$. As the speed of the vortex $c_{v}=2,6,10$ increases the displacement thickness approaches that given by the asymptotic result $c_{v} \rightarrow \infty$ for which the maximum thickness is at $X=-1$ / $\sqrt{3}$. Note that as $c_{v}$ decreases the maximum amplitude of (a) vortices of positive strength $\kappa=\frac{1}{2}$ increases faster than that for (b) vortices of negative strength $\kappa=-\frac{1}{2}$. This suggests that the steady solution will break down sooner-at a larger value of $c_{v}$-for $\kappa=\frac{1}{2}$ than for $\kappa=-\frac{1}{2}$; see Sec. III. (b) For the caption see the previous figure.

ment thickness can be seen to approach that given by the asymptotic result.

\section{DISCUSSION}

A steady solution to the boundary layer equations disturbed by the passage of a fast vortex has been presented. In the limit of large vortex velocity $\left(c_{v} \rightarrow \infty\right)$, the boundary layer is weak and an asymptotic solution is found. The weak boundary layer approximation of Sec. II A suggests that as $c_{v}$ is increased through finite values of $c_{v}$, the results should converge to the asymptotic results. Our numerical results of 
streamfunction, stagnation point location, shear stress, and displacement thickness do indeed confirm this.

Moreover, our numerical results demonstrate the existence of steady solutions for a fast vortex for at least $c_{v} \geqslant 1$. This result can be compared qualitatively to that of Degani and Walker, ${ }^{9}$ who find that separation on a rotating and translating cylinder is suppressed when $\Omega a / U_{0}>1.9$, where $\Omega$ is the angular speed of rotation, $a$ is the cylinder radius, and $U_{0}$ is the speed of translation. In general, we believe that if the relative wall speed is large compared to the external velocity field, then eruption of the boundary layer can be suppressed. Physically, this can be explained by noting that the effects of the external velocity field are advected away by the moving boundary before they can accumulate into a temporal eruption.

The breakdown of the existence of the steady solution is associated with local flow reversal in the frame of the vortex. It may be possible to make analytical progress in the understanding of finite-time singularity formation by considering the manner in which the steady solution fails as $c_{v}$ decreases. Several problems of practical interest that can be modeled as a vortex-boundary layer interaction have been discussed in the literature. For example, the interactions of a wall with hairpin vortices, trailing vortices, and other shed vortical structures are relevant to the performance of both aircraft and marine vessels. Often, performance is limited by the occurrence of boundary layer eruption. The demonstration here of a steady solution suggests that if the vortex is advected sufficiently quickly over the boundary then eruption may be suppressed.

\section{ACKNOWLEDGMENTS}

The authors are grateful to the National Science Foundation who sponsored this work under Grant No. CTS-
9206828. A NATO Collaborative Research Grant has allowed S.L. to maintain fruitful contact with Professor G. J. F. van Heijst, University of Eindhoven, the Netherlands. We would also like to thank Mr. Chih-Yu Lin, who donated his time to help format the presentation of the results.

${ }^{1}$ T. L. Doligalski and J. D. A. Walker, "The boundary layer induced by a convected two-dimensional vortex," J. Fluid Mech. 139, 1 (1984).

${ }^{2}$ J. D. A. Walker, "The boundary layer due to rectilinear vortex," Proc. R. Soc. London Ser. A 359, 167 (1978).

${ }^{3}$ J. W. Elliott, F. T. Smith, and S. J. Cowley, "Breakdown of boundary layers: (i) on moving surfaces; (ii) in semi-similar unsteady flow; (iii) in fully unsteady flow," Geophys. Astrophys. Fluid Dyn. 25, 77 (1983).

${ }^{4}$ V. J. Peridier, F. T. Smith, and J. D. A. Walker, "Vortex-induced boundarylayer separation. Part 1. The unsteady limit problem $\mathrm{Re} \rightarrow \infty$," J. Fluid Mech. 232, 99 (1991).

${ }^{5}$ E. C. Adams, F. T. Smith, and A. T. Conlisk, "An adaptive grid scheme for the unsteady boundary layer induced by a vortex," AIAA Paper No. 942294, 1994.

${ }^{6}$ T. L. Doligalski, C. R. Smith, and J. D. A. Walker, "Production mechanism for turbulent boundary-layer flows," Prog. Astronaut. Aeronaut. 72, 47 (1980).

${ }^{7}$ T. L. Doligalski, C. R. Smith, and J. D. A. Walker, "Vortex interactions with walls," in Annual Review of Fluid Mechanics, edited by J. L. Lumley, M. van Dyke, and H. L. Reed (Annual Reviews, Palo Alto, CA, 1994), pp. 573-616.

${ }^{8}$ J. A. Luton, S. A. Ragab, and D. P. Telionis, "Interaction of spanwise vortices with a boundary layer," AIAA Paper No. 94-2377, 1994.

${ }^{9}$ A. T. Degani and J. D. A. Walker, "Calculation of unsteady separation from stationary and moving walls," in Bluff-Body Wakes, Dynamics and Instabilities, IUTAM Symposium, Gottingen, Germany, 1992, pp. 23-26.

${ }^{10}$ G. F. Carrier and C. E. Pearson, Partial Differential Equations: Theory and Technique (Academic Press, New York, 1976), p. 25.

${ }^{11} \mathrm{~F}$. G. Blottner, "Investigation of some finite-difference techniques for solving the boundary layer equations," Comput. Methods Appl. Mech. Eng. 6, 1 (1975). 